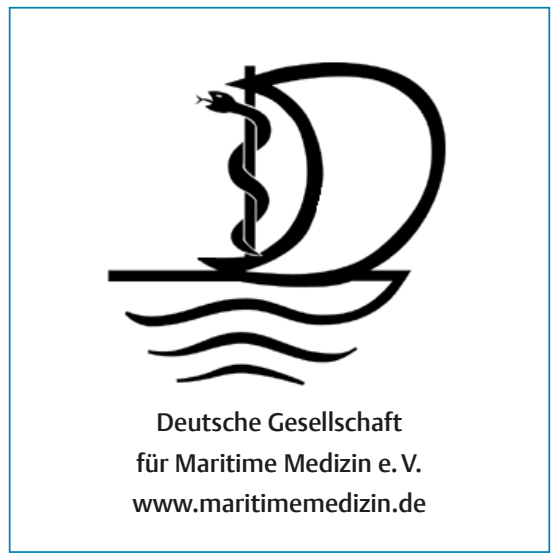

\title{
Liebe Mitglieder,
}

wir stehen vor der Sommerpause und dennoch steckt der Vorstand ,bis über beide Ohren' in den Vorbereitungsarbeiten für unsere Jahrestagung im Oktober. Wir feiern dann ja unser 25-jähriges Jubiläum und es ist unser Ehrgeiz, Ihnen ein besonders attraktives Programm zu bieten. Das Ergebnis finden Sie weiter unten und ich hoffe, dass unsere Mühen durch Ihre zahlreiche Teilnahme belohnt werden!

Einige Mühe macht uns derzeit auch noch unsere Internetseite. Ihnen ist sicher aufgefallen, dass sie im zweiten Quartal etwas hinter die Zeit geraten ist. Wenn Sie diese Zeilen lesen, ist das Problem hoffentlich gelöst und wir sind mit unserem neuen Content-Management-System flexibler und aktueller als zuvor.

Viel Mühe machte uns - nach Wechsel einiger Vorstandsposten - die Ingebrauchnahme unserer Mitgliederverwaltungssoftware. Unter Anleitung von Stefan Neidhardt haben Sekretär, Schatzmeister und ich aber mit den Werkzeugen einige Sicherheit erreicht und wir können nun auch hier flexibler und aktueller sein! Nicht wenige Mitglieder haben bemerkt, dass wir noch einmal zu ihren Daten gefragt haben und als Erfolg haben wir jetzt eine 86\%-Rate hinsichtlich der Möglichkeit, mit Ihnen per Email in Verbindung zu treten!

Das SEPA-Einzugsverfahren für die Mitgliederbeiträge liegt derzeit bei einer Erfolgsquote von 74\%. Alle Mitglieder, die bisher ihre Email noch nicht bekannt gegeben haben, beziehungsweise noch keine SEPA-Einzugsermächtigung erteilt haben, wurden angeschrieben und ich bitte herzlich um eine baldige Rückantwort!

Zwei große Kongresse liegen hinter uns. Zum einen der Kongress der International Maritime Rescue Federation, der aus Anlass des 150-jährigen Jubiläums der DGzRS in Bremerhaven stattfand (siehe den Bericht unseres Mitglieds Denis Gümbel) und das $13^{\text {th }}$ International Symposium on Maritime Health in Bergen/ Norwegen (siehe Bericht unseres Mitglieds Bernd Schepers).

Mit dem Blick voraus auf unsere

Jahrestagung verbleibe ich mit den besten Grüßen

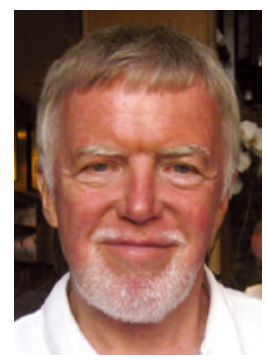

Ihr

Klaus Seidenstücker

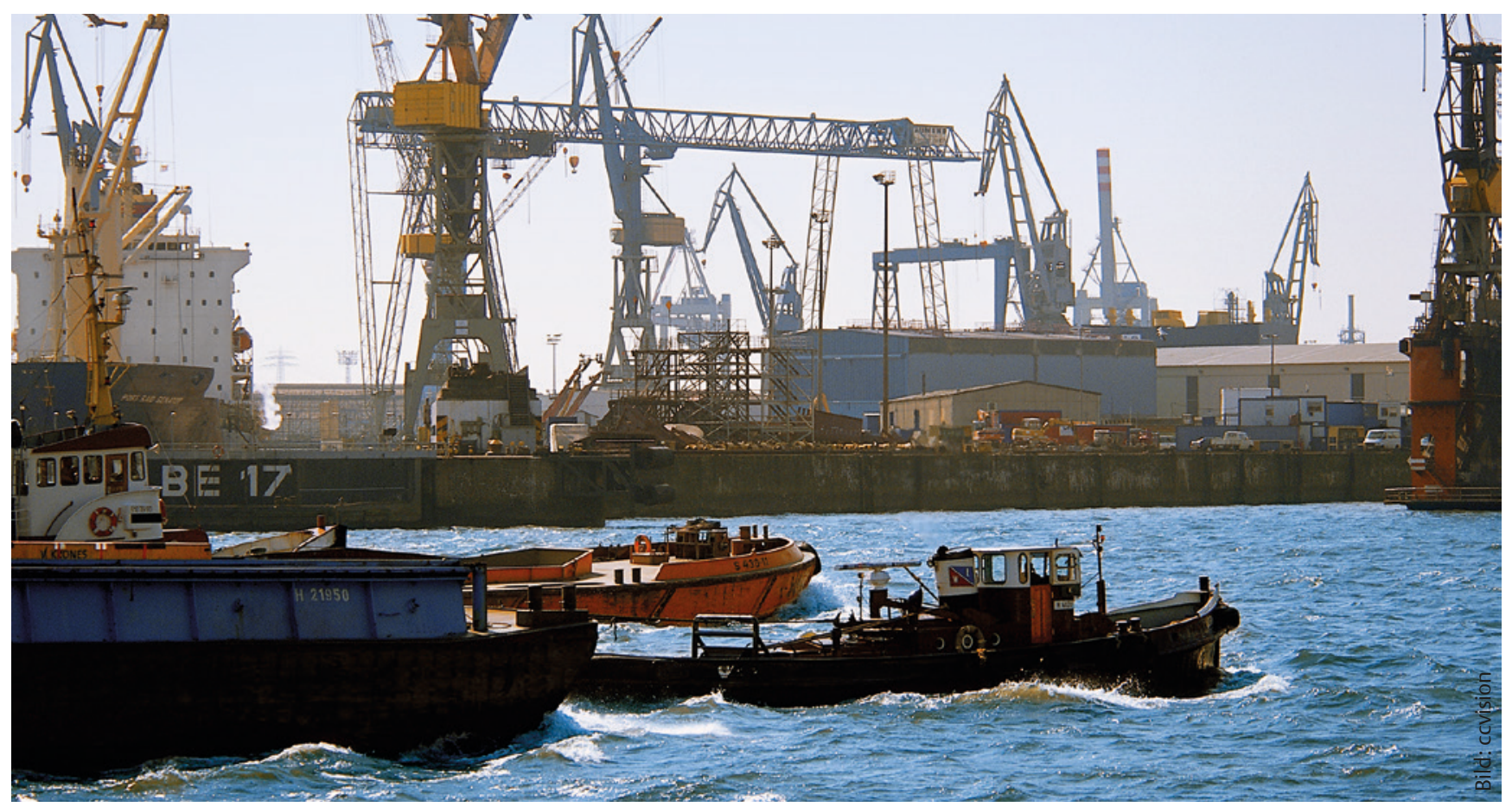




\section{Symposium Maritime Medizin}

aus Anlass des 25-jährigen Jubiläums der Deutschen Gesellschaft für Maritime Medizin e.V. am Sonnabend, den 10. Oktober 2015 in Hamburg; Empire Riverside Hotel, Bernhard-Nocht-Str. 97

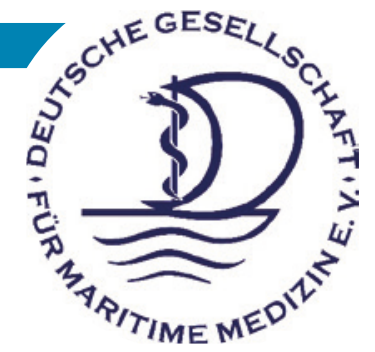

08:30- Registrierung

09:30

09:30 Begrüßung

Klaus-Herbert Seidenstücker, Hamburg

09:35 Seemannsandacht

Jan Oltmanns, Hamburg

09:45 Keynote Lecture: Seafarer health: who cares? Can historical studies inform current policy and practice?

Tim Carter, London/Bergen

10:30 Kaffeepause

10:45 Themenblock I -

Maritime Forschung, Lehre, Ausbildung

Volker Harth, Hamburg

Todesursachen von Seeleuten

und Kreuzfahrtpassagieren

Marcus Oldenburg, Hamburg

Seekrankheit - Symptomatik, Medikation und Dienstfähigkeit

Andreas Koch, Kronshagen

Das First-Responder-Training der Marine ein Modell für die zivile Seefahrt?

Dirk Chlustin, Neustadt

Medizinische Ausbildung nautischer Offiziere Julia Knopp, Rostock

12:30 Buffet - Besuch der Aussteller
13:30 Themenblock II -

Prävention im maritimen Umfeld

Bernd-Fred Schepers, Hamburg

Seuchenschutz in deutschen Häfen

Martin Dirksen-Fischer, Hamburg

Ebola, Malaria, Bettwanzen \& Co

Was macht Sinn im Infektionsschutz für Seeleute?

Clara Schlaich, Hamburg

Trinkwasserhygiene auf Offshoreanlagen

Christoph Sevenich, Kiel

Offshoretauglichkeit im internationalen Vergleich Alexandra Preisser, Hamburg

15:00 Kaffeepause

15:30 Themenblock III -

Kurative Medizin auf See

Olaf Schedler, Bad Saarow

Damage Control Surgery - gibt es Anwendungsmöglichkeiten für die zivile Schifffahrt?

Wilm Rost, Hamburg

Medizinische Vorfälle in deutschen Offshorewindparks - Gefährdungspotenziale, Luftrettung und Präventionsmaßnahmen

Dirk Dethleff, Hamburg

Erfahrungen deutscher Reedereien mit der Flüchtlingsrettung im Mittelmeer

Marcus Oldenburg, Hamburg

Erste Erfahrung der Marine mit Bootsflüchtlingen Wolfgang Toepfer, Rostock

17:00 Ende der Veranstaltung

Die Ärztekammer Hamburg hat die Veranstaltung mit 6 Fortbildungspunkten zertifiziert.

Die Publikation eines Abstractbandes erfolgt auf der Internetseite der DGMM e.V.

Die Teilnahmegebühr beträgt für Mitglieder der DGMM e.V. 50Euro und für Nichtmitglieder 75 Euro.

Anmeldung über die Internetseite www.maritimemedizin.de/aktuelles 


\section{Neu in unserem Vorstand: Alexandra Preisser}

Als gebürtiger Frankfurterin (am Main) war Alexandra Preisser die maritime Medizin keineswegs in die Wiege gelegt. Früh begann sie dennoch in der Segeljolle „Optimist“" Lee und Luv zu unterscheiden. 1982 begann sie ihr Studium in Marburg an der Lahn, nutzte in dieser Zeit das universitätseigene Bootshaus am Edersee in Nordhessen als Übungsleiterin im Jollensegeln und begann 1989 mit dem Segeln auf Jachten. Der Abschluss von BK-Zeugnis und dem amtlichen Sportseeschifferschein berechtigten sie als Jachtskipperin tätig zu sein, welches sie in Urlaubsreisen auf Ostsee und Mittelmeer gerne wahrnahm.

Nach dem Studium zog es sie im Rahmen ihrer Weiterbildung zur Fachärztin für Innere Medizin nach Station in Bad Soden am Taunus gen Norden. Nach einigen Mo- naten in Reinbek (bei Hamburg) arbeitete sie schließlich - bis zu dessen Schließung 1997 - im Hamburger Hafenkrankenhaus! Nun war wirklich Salz in der Luft!

Von 1997 bis 2001 absolvierte Alexandra Preisser die Weiterbildung im Schwerpunkt Pneumonologie und erwarb die Zusatzbezeichnung Allergologie in der Lungenabteilung des AK HamburgHarburg.

Nach Intermezzo in einer kardiologischen Praxis folgte 2002 bis 2005 die Weiterbildung zur Ärztin für Arbeitsmedizin am Zentralinstitut für Arbeitsmedizin in Hamburg; damals noch vergesellschaftet mit dem Hamburg Port Health Center und da war die Exposition gegenüber der
Berufsschifffahrt unvermeidlich.

Seit 2006 ist Alexandra Preisser wissenschaftliche Mitarbeiterin am Universitätsklinikum Hamburg Eppendorf (UKE) und Leiterin der Arbeitsgruppe ,Klinische Arbeitsmedizin' am Zentralinstitut für Arbeitsmedizin und Maritime Medizin (ZfAM) in Hamburg. Seit Februar dieses Jahres ist sie Oberärztin am UKE und stellvertretende Leiterin des ZfAM.

Alexandra Preisser ist Mitglied unserer Gesellschaft seit 2002.

Sie ist Sprecherin unserer Arbeitsgruppe Eignungsuntersuchungen für Offshore Arbeitnehmer seit 2011 und seit Januar dieses Jahres als Beisitzerin in unserem Vorstand.

\section{Jan Gerd Hagelstein - unser Kassenprüfer schreibt maritimmedizinische Geschichte!}

Jan Gerd Hagelstein erblickte 1957 in Bogotá, Kolumbien, das Licht dieser Welt.

Seine berufliche Laufbahn begann mit einer Lehre als Schifffahrtskaufmann. Erst danach entschloss er sich für die Medizin und führte 1990 diese beiden Lebenselemente zusammen, in-

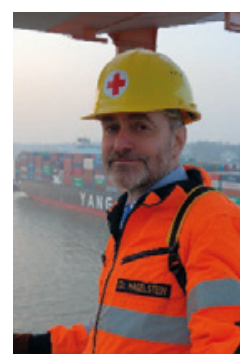

1995 war es mit der aktiven Zeit in der Bundeswehr vorbei und Jan Gerd Hagelstein machte als Allgemeinmediziner in Hamburg fest. Mit seiner Praxis spezialisierte er sich von Beginn an auf die Versorgung der Seeleute zunächst an seinem Praxissitz in der Hamburger Neustadt, dann am Wilhelmsburger Krankendem er sich zur Marine meldete. Jan Gerd Hagelstein fuhr zunächst als Geschwaderarzt zur See, was sein Interesse für die Taucherei weckte. Er absolvierte den großen Taucherarztlehrgang am Schifffahrtmedizinischen Institut der Marine in Kronshagen und Neustadt.

1993 übernahm er als Chef die MarineSanitätsstaffel in Glückstadt. haus Groß Sand.

2010 promovierte er mit dem Thema ,Schwere Seeunfälle mit Personenschäden in der Kauffahrteischifffahrt".

Seit 2015 gehört seine Seemannsambulanz als Fachabteilung zur Klinik, in der Hagelstein nun die Position eines Chefarztes innehat. Damit gibt es nach gut 10-jäh- riger Unterbrechung wieder eine Art Hafenkrankenhaus in Hamburg!

In seiner Freizeit sitzt Jan Gerd Hagelstein unter anderem ehrenamtlich mit am Vorstandstisch der Deutschen Seemannsmission Hamburg-Harburg, welche den bekannten internationalen Seemannsclub ,Duckdalben' und die ,seafarer's lounges“ der Kreuzfahrtterminals bereedert.

Jan Gerd Hagelstein ist Mitglied unserer Gesellschaft seit 1992 und unser Kassenprüfer über mehr als 4 Wahlperioden.

Allen Absolventen des Kompaktkurses Maritime Medizin ist er durch seine launigen Vorträge und die von großer Empathie gekennzeichneten Einblicke in seinen Praxisalltag in lebhafter Erinnerung. 


\section{Internationaler maritimmedizinischer Kongress in Norwegen}

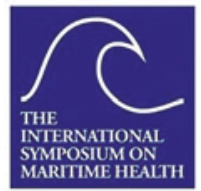

Vom 23. bis 26. Juni 2015 fand das $13^{\text {th }}$ International Symposium on Maritime Health in Bergen, Norwegen, statt diesmal in organisatorischer Verantwortung des Norwegian Centre for Maritime Medicine und wie immer unter der Schirmherrschaft der International Maritime Health Association (IMHA).

Nach der Eröffnungszeremonie mit musikalischer Umrahmung im Radisson Blue Royal Bryggen Hotel mit Grußansprachen von Vertretern der Norwegian Maritime Authority, der International Labour Organisation, der International Maritime Organization,der International Transport Worker's Federation, der Bergen Shipowner's Association, der Norwegian Association of Maritime Medicine, des ITF-Seafarer's Trust sowie des Präsidenten der IMHA folgte ein Empfang des Bergener Bürgermeisters in der historischen Hakonshalle und die Teilnahme am Mittsommernachtsfeuer.

Healthy Seafarers -

Healthy Shipping

Vom 24. bis 26. Juni wurden unter dem Leitmotiv „Healthy Seafarers - Healthy Shipping“" rund 70 Vorträge gehalten und eine Postersession gezeigt. Die Vorträge fanden zum Teil in Parallelveranstaltungen in verschiedenen Sälen des Tagungshotels statt. Themenblöcke waren unter anderen:

- Healthy Shipping

- Infectious Diseases

- Development in Remote Care

- Occupational Risks to Health

- Physical Demands at Sea and their Measurements

- Telemedical Maritime Assistance Service/TMAS

- Wellbeing, Gender and Culture

- Healthy Seafarers and Healthy Passengers

- Maritime Medical Practice and Care at Sea

- Risk Management at Sea

- Chronic Diseases in Seafarers

- Perspectives on Maritime Health

- Healthy Fisheries

- The Cruise Sector

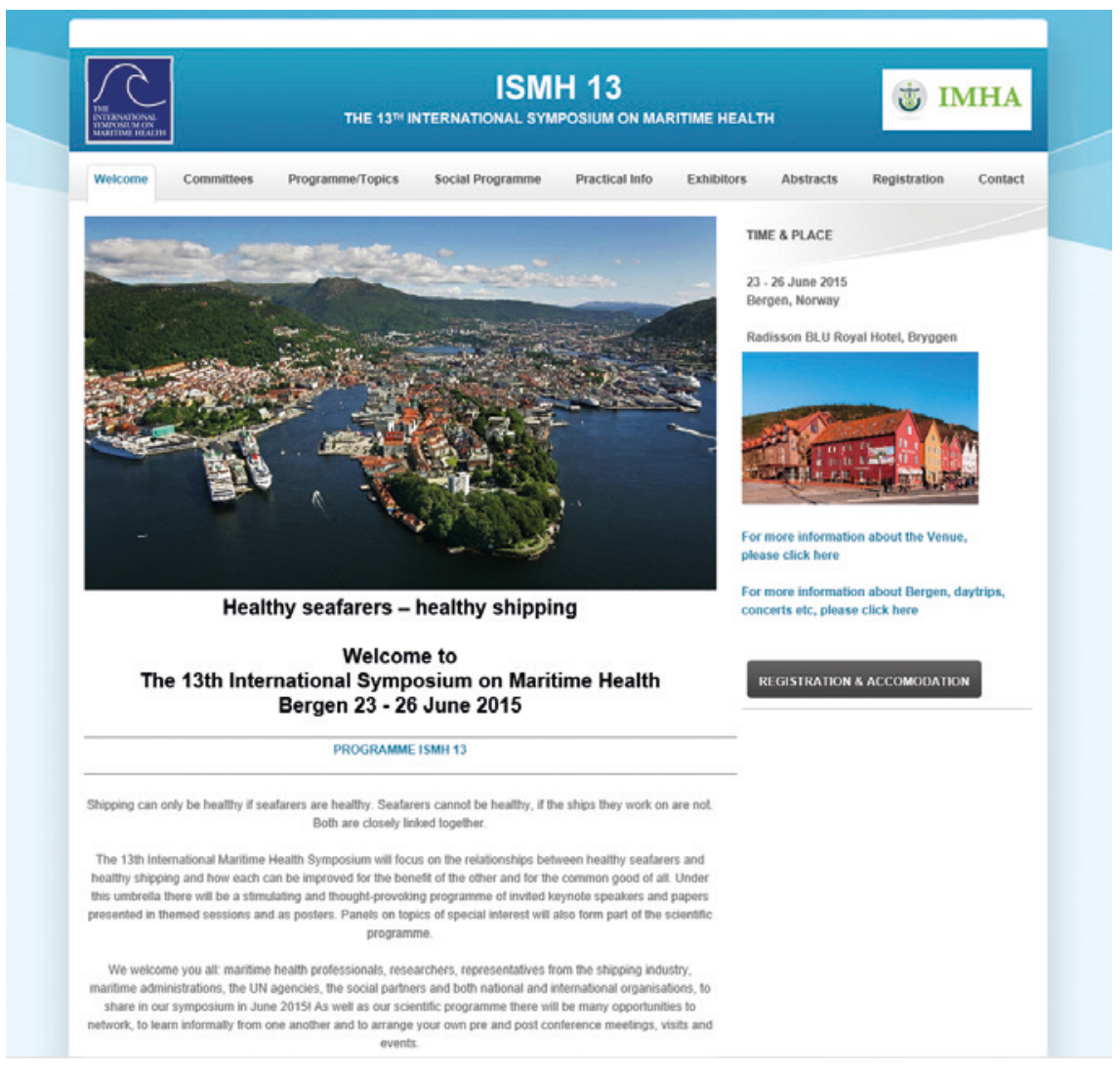

Die lange Liste der Vorträge und Poster, der verschiedenen Leitthemen sowie die immer wieder eingeschobenen Diskussionen machten erneut die Vielfalt der maritimmedizinischen Themen deutlich. Der teilweise unterschiedliche Umgang mit den praktischen Fragestellungen in den verschiedenen Schifffahrtsländern weltweit offenbarte außerdem, wie wichtig weiterer intensiver Erfahrungsaustausch und weitere internationale Harmonisierung auf diversen maritimmedizinischen Gebieten sind und welch erheblicher Forschungsbedarf für die wissenschaftlichen Institutionen besteht.

\section{Deutsche Seite gut vertreten}

Unter den 184 gelisteten Teilnehmern war die deutsche Seite mit 19 Teilnehmern - überwiegend DGMM-Mitglieder - erfreulich gut vertreten, von denen insgesamt 10 Vorträge sowie mehrere Poster in das Symposium eingebracht wurden.

\section{Mitgliederversammlung der IMHA}

Eingebettet in das Symposium war wie immer die Mitgliederversammlung der
IMHA mit den Neuwahlen für den Vorstand. Gewählt wurden:

- Präsidentin: Dr. Ilona Denisenko, Russland

- Vize-Präsident: Dr. Joseph Abesamis, Philippinen

- Sowie die Vorstandsmitglieder: Dr. David Dungan, Südafrika, Dr. Suresh Idnani, Indien, Dr. Nicholas Ioannidis, Griechenland, Dr. Cornelia Pattiasina, Indonesien, Dr. Klaus Seidenstücker, Deutschland.

Für die erfolgreiche Durchführung des Symposiums mit einem ebenfalls beeindruckenden und unvergesslichen Rahmenprogramm erhielten die Veranstalter und alle Mitwirkenden großes Lob, Dank und Anerkennung.

Das $14^{\text {th }}$ International Symposium on Maritime Health findet vom 21. bis 24. März 2017 in Manila, Philippinen, statt. Für das Folgesymposium 2019 ist von deutscher Seite (DGMM und ZfAM) eine Bewerbung für Hamburg geplant.

Dr. Bernd-Fred Schepers, Hamburg 


\section{Internationaler Kongress für Rettungsorganisationen}

\section{in Bremerhaven}

Nach einem spektakulären Wochenende im Rahmen der 150-Jahrfeier der Deutschen Gesellschaft zur Rettung Schiffbrüchiger (DGzRS) für die breite Öffentlichkeit mit Paraden, Ausstellungen und begleitenden Veranstaltungen mit der Beteiligung von Search-and-RescueSchiffen (SAR) aus verschiedenen Ländern, fand vom 1. bis 4. Juni 2015 der World Maritime Rescue Congress (WMRC 2015) der International Maritime Rescue Federation (IMRF) in Bremerhaven statt. Insgesamt zählte der Kongress 639 Teilnehmer aus über 22 Ländern. Mehr als 60 internationale SAR-Institutionen waren vertreten.

Die Workshops und Präsentationen aller 3 Kongresstage waren in 3 Themenkomplexe gegliedert:

- SAR Organisation and Management

- SAR Coordination

- SAR Operations

Besonderer Fokus wurde auf Prävention, Ausbildung und Training gelegt. Die Mass Rescue Operations (MRO) wurden als „low probability, but high consequence events“ identifiziert und werden auch im Hinblick der steigenden Zahl

solcher Ereignisse in $\mathrm{Zu}$ kunft verstärkt im Fokus internationaler Rettungsorganisationen sein.

Gefahrenabwehr bei

Katastrophen

Um eine kontinuierliche Verbesserung der Gefahrenabwehr bei Großschadensereignissen auf See zu erreichen, wurde das MROProjekt der IMRF gestartet.

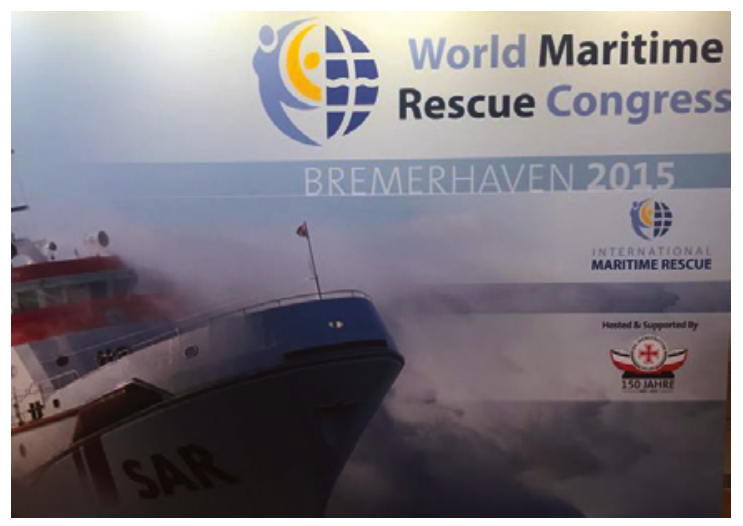
Während SAR-Dienste in der Mehrzahl der Fälle „einfache und komplexe (Einzel-) Schadenslagen“ bewältigen, sind „Katastrophen“ seltene Ereignisse. Wenn jedoch eine Katastrophe eingetreten ist, hat sie enorme Auswirkungen, so David Jardine-Smith von der IMRF. Das MRO-Projekt fördert durch Sensibilisierung aller Beteiligten, Identifikation von Problemen bei stattgehabten Ereignissen, Verbesserung von Leitlinien, eine frei zugängliche Datenbank mit einer Präsenzbibliothek und internationalen Workshops die internationale Zusammenarbeit und Expertise.

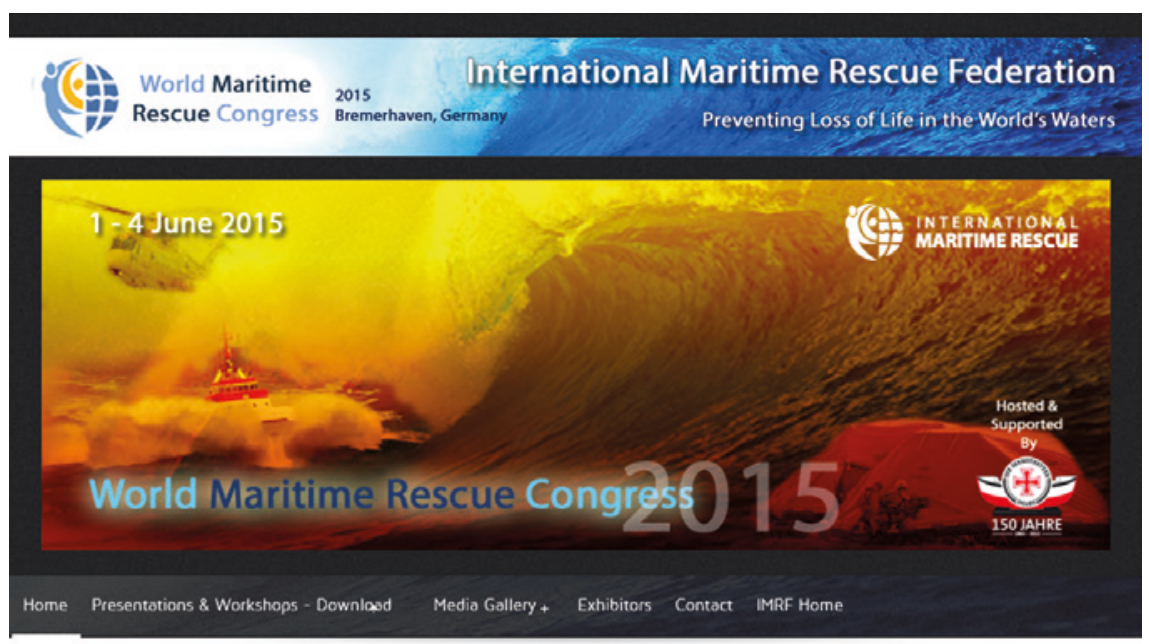

Home

$\Theta$ Print $\cong$ Email

World Maritime Rescue Congress \& Exhibition 2015

The IMRF Congress is a true conference. It is designed to enable people from search and rescue (SAR) services all around the world to talk together, to confer, about a wide range of subjects of vital importance to them and to the people they seek to save.
Translate this Website

is Select Language

Follow Us - WMRC
Umfangreiches Kongressprogramm

Neben vielen technischen Aspekten der Seerettung, die im Rahmen von Schadensereignissen auf See von den SAROrganisationen jeden Tag bewältigt werden, steht die Rettung und die medizinische Versorgung der Verletzten und Erkrankten im Vordergrund aller Bemühungen. Während der Workshops wurden medizinisch relevante internationale Forschungsprojekte, Forschungsergebnisse und Innovationen präsentiert und diskutiert: Wassersicherheitstrainings für Kinder und Prävention von Ertrinkungsunfällen, Einsatz von Drohnen, Flüchtlingshilfe, Maritime Simulatorübungen (marSimnet, Mona Lisa), stress exposure training, Fundraising sind nur einige davon.

Neben einer informativen Industrieausstellung gab es darüber hinaus Gelegenheit zum Erfahrungsaustausch mit Einsatzkräften internationaler SAR-Dienste und zur Besichtigung hochmoderner SAR-Rettungsschiffe.

Am Ende des Kongresses wurde Kapitän Udo Fox (DGzRS) als neuer Vorsitzender der IMRF für die kommenden 8 Jahre gewählt.

Einen Überblick über die thematische Bandbreite des gesamten Kongresses, einschließlich Downloadmöglichkeit der Präsentationen, gibt die Kongressseite www.international-maritime-rescue. org/index.php/homewmrc.

Dr. Denis Gümbel, Caspar Ottersbach Universitätsmedizin Greifswald Abt. für Unfallund Wiederherstellungschirurgie, Projekt KOMPASS 